\title{
A SPATIALLY ADAPTIVE POISSONIAN IMAGE DEBLURRING
}

\author{
Alessandro Foi ${ }^{a b}$, Sakari Alenius ${ }^{c}$, Mejdi Trimeche ${ }^{c}$, Vladimir Katkovnik $^{a *}$ and Karen Egiazarian ${ }^{a}$ \\ ${ }^{a}$ Institute of Signal Processing, Tampere University of Technology, Tampere, Finland \\ ${ }^{b}$ Dipartimento di Matematica, Politecnico di Milano, Italy \\ ${ }^{c}$ Nokia Research Center, Tampere, Finland
}

\begin{abstract}
A spatially adaptive image deblurring algorithm is presented for Poisson observations. It adapts to the unknown image smoothness by using local polynomial approximation $(L P A)$ kernel estimates of varying scale and direction based on the intersection of confidence intervals $(I C I)$ rule. The signal-dependant characteristics of the Poissonian noise are exploited to accurately compute the pointwise variances of the directional estimates. The results show that this accurate pointwise adaptive algorithm significantly improves the image restoration quality.
\end{abstract}

\section{INTRODUCTION}

In many imaging systems the recorded observations have the physical meaning of numbers of detected photons. The photons are counted at different spatial locations and in this way form an image of an object. This sort of scenario is typical for many imaging problems in medicine, including positron and single-photon emission tomography, in gamma astronomy, microscopy, and photonlimited optical imaging. The Poisson distribution is the conventional probabilistic model for the random number of photons detected during an exposure time. An important consumer application where Poissonian distributions dominate are the widespread CCD/CMOS-sensor digital cameras (e.g. [13]).

An optical blurring is typically introduced into the observation process. This distortion of the image is commonly modeled by the convolution $(y \circledast v)(x)$ of the true image $y$ with the pointspread function (PSF) $v$ of the optical system. It is assumed that the observations $z(x)$ are Poissonian, according to the model

$$
z(x) \sim \mathcal{P}((y \circledast v)(x)),
$$

where $\mathcal{P}$ denotes the Poisson distribution. This model means that $E\{z(x)\}=(y \circledast v)(x)$ and $\sigma_{z}^{2}(x)=\operatorname{var}\{z(x)\}=(y \circledast v)(x)$. Thus, the observation variance $\sigma_{z}^{2}(x)$ is signal dependent and, consequently, spatially variant. In our approach we make explicit use of this variance function to reconstruct the image $y$ from the noisy observations $z$. Observe that (1) can be rewritten in the additive form $z(x)=(y \circledast v)(x)+\eta(x)$, where the noise term $\eta(x)$ has zero mean and variance $\sigma_{\eta}^{2}(x)=(y \circledast v)(x)$.

\subsection{Maximum likelihood $(M L)$ inverse}

Since the random observation $z$ has a Poisson distribution with the mean $E\{z(x)\}=(y \circledast v)(x)$, the corresponding log-likelihood is

$$
L=\log (l)=\sum_{s}\left[-(y \circledast v)\left(x_{s}\right)+z_{s} \log \left((y \circledast v)\left(x_{s}\right)\right)\right],
$$

${ }^{*}$ The work of Dr. Katkovnik was supported in part by a Visiting Fellow grant from Nokia Foundation. where the term $\log \left(z_{s} !\right)$ independent of $y$ is omitted. Then, the maximum-likelihood gives the estimate of $y$ as $\hat{y}(x)=\max _{y} L$.

The solution of the inverse Poisson problem can be obtained using the recursive Richardson-Lucy algorithm [1]. Usually the problem is ill-conditioned and the solution is unacceptably noisy. Different regularization or damping techniques are used to improve the reconstruction (e.g. [12],[14]).

\subsection{Local $M L$ Poisson inverse}

A local version of the likelihood is different from (2) by the window function $w_{h}(x)=w(x / h)$ and can be presented in the form

$L_{h}=\sum_{s}\left[-(y \circledast v)\left(X_{s}\right)+z_{s} \log \left((y \circledast v)\left(X_{s}\right)\right)\right] w_{h}\left(x-X_{s}\right)$, where the scale parameter $h$ defines the degree of localization of the likelihood [2]. Assuming further that a locally constant model is used for the convolution $y \circledast v$, we arrive to

$$
L_{h}(x, C)=\sum_{s}\left(-C+z_{s} \log C\right) w_{h}\left(x-X_{s}\right) .
$$

Then the $M L$ estimate of $C$ has the form

$$
\hat{C}_{h}(x)=\left(z \circledast g_{h}\right)(x), \quad g_{h}(x)=w_{h}(x) / \sum_{\xi} w_{h}(\xi),
$$

where $\hat{C}_{h}(x)$ is the nonparametric Nadaraya-Watson estimate for $(y \circledast v)(x)$. If $\hat{C}_{h}(x)$ is found, the estimate of $y$ (with notation $\hat{y}_{h}$ ) is a solution of the linear equation

$$
\left(\hat{y}_{h} \circledast v\right)(x)=\hat{C}_{h}(x) .
$$

In the frequency domain, using capital letters for the Fourier transform $\mathcal{F}$ of the corresponding variables, it gives

$$
V(f) \hat{Y}_{h}(f)=G_{h}(f) Z(f) .
$$

Thus, we arrive to the linear inverse problem having as an input the estimate $\hat{C}_{h}(x)$. The unknown $\hat{Y}_{h}(f)$ is a solution of the linear system (4). Because ill-conditioning ( $Z$ is noisy), this sort of systems is commonly solved by regularization, as $\hat{Y}_{h}(f)=$ $V(-f) G_{h}(f) Z(f) /\left(|V(f)|^{2}+\varepsilon^{2}\right)$. Remind that the conditioning of (4) is defined by the convolution kernel $g_{h}$. In general, depending on the local smoothness of the original data $y$ and the local variance of the noise $\eta$, different values of scale parameter $h$ may be appropriate for different regions of the image. Our algorithm described further uses $L P A$ [2] kernels $g_{h}$ to filter the observations and is based on application of the $I C I[6,7]$ directly to the estimate $\hat{y}_{h}$ in order to select a pointwise-adaptive value $h^{+}$of the scale $h$.

We remark that, mostly because of their lower complexity and good stability, also linear Wiener filters have been used extensively for the restoration of blurred images with Poissonian and, more generally, signal-dependant noise (e.g. [3],[11]). 


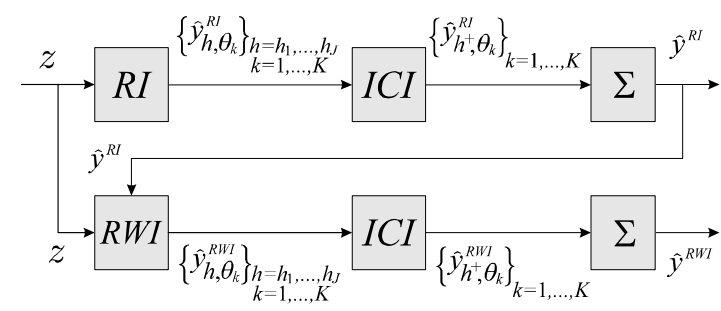

Fig. 1. Directional $L P A-I C I$ regularized Wiener inverse algorithm. In the first line of the flowchart the $R I$ estimates are calculated for a set of scales and directions, the ICI is used to obtain the pointwise optimal scale directional estimates that are then fused into the $\hat{y}^{R I}$ estimate. In the second line the $R W I$ estimates are calculated using $\hat{y}^{R I}$ as a reference signal in Wiener filtering, again $I C I$ and fusing are performed to obtain the final $\hat{y}^{R W I}$ estimate.

\section{LINEAR INVERSE WITH DIRECTIONAL ADAPTIVE LPA-ICI FILTERING}

This algorithm uses the non-parametric regularized inverse $(R I)$ and regularized Wiener inverse (RWI) LPA-ICI deconvolutions developed for the Gaussian inverse in [8],[9] and for inverse halftoning (colored noise) in [5]. We briefly review here the general outline of the original algorithm; details of its implementation for the Poissonian case are given in the following subsections.

The $R I-R W I$ algorithm is a two stages procedure where the final estimate of $y$ is given by the $R W I$ deconvolution scheme that uses the regularized inverse $(R I)$ estimate as a reference signal. In both steps the directional $L P A-I C I[9,4]$ technique is exploited in order to construct in a pointwise manner an anisotropic nonparemetric estimate of the signal. The algorithm is illustrated in Figure 1. For the AWGN case, $\eta \sim \mathcal{N}\left(0, \sigma^{2}\right)$, the filtering was performed completely in the Fourier domain as

$$
\begin{aligned}
& \hat{Y}_{h, \theta}^{R I}(f)=\frac{V(-f) G_{h, \theta}(f)}{|V(f)|^{2}+\varepsilon_{1}^{2}} Z(f), \\
& \hat{Y}_{h, \theta}^{R W I}(f)=\frac{V(-f)|Y(f)|^{2} G_{h, \theta}(f)}{|V(f) Y(f)|^{2}+\varepsilon_{2}^{2} \sigma^{2}} Z(f), \quad(R W I),
\end{aligned}
$$

where $\varepsilon_{1}, \varepsilon_{2}>0$ are regularization parameters. The adaptive procedure assumes that the estimates $\left\{\hat{y}_{h, \theta_{k}}^{R I}\right\}_{h \in H}$ are calculated according to (5) for a set of scales $H$ and a number of directions $\left\{\theta_{k}\right\}_{k=1}^{K}$ and the ICI rule selects the best scales for each direction and for each pixel. The $G_{h, \theta}$ in the above formulas correspond to a collection of directional varying scale $L P A$ kernels $g_{h, \theta}$. In this way we obtain the directional adaptive-scale estimates $\hat{y}_{h^{+}}^{R I}{ }_{\left(x, \theta_{k}\right), \theta_{k}}, k=1, \ldots, K$, which are fused by a convex combination into the final one $\hat{y}^{R I}$. This $\hat{y}^{R I}$ serves as the reference signal, instead of $y$, in the $R W I$ procedure (6) (see Figure 1). The adaptive $R W I$ algorithm is similar and gives the $I C I$ adaptive-scale estimates $\hat{y}_{h^{+}\left(x, \theta_{k}\right), \theta_{k}}$ for each direction and $x$. Then, the final anisotropic estimate $\hat{y}^{R W I}$ is obtained again by a convex fusing of these directional estimates.

Let us briefly remind the $I C I$ optimal scale selection rule [6, 7]. Given a set of varying scale kernel estimates $\left\{\hat{y}_{h_{j}}(x)\right\}_{j=1}^{J}$ with increasing scale, we determine a sequence of confidence intervals $\mathcal{D}_{j}=\left[\hat{y}_{h_{j}}(x)-\Gamma \sigma_{\hat{y}_{h_{j}}}, \hat{y}_{h_{j}}(x)+\Gamma \sigma_{\hat{y}_{h_{j}}}\right]$, where $\Gamma>0$ is a threshold parameter. The ICI rule can be stated as follows: Consider the intersection of confidence intervals $\mathcal{I}_{j}=\bigcap_{i=1}^{j} \mathcal{D}_{i}$ and let $j^{+}$be the largest of the indexes $j$ for which $\mathcal{I}_{j}$ is non-empty, $\mathcal{I}_{j+} \neq \varnothing$ and
$\mathcal{I}_{j++1}=\varnothing$. Then, the adaptive scale $h^{+}$is defined as $h^{+}=h_{j+}$ and the adaptive-scale kernel estimate is $\hat{y}_{h^{+}}(x)$.

We remark that the use of the ICI rule requires the calculation of the standard deviations of the individual varying scale directional estimates $\left\{\hat{y}_{h, \theta_{k}}^{R I}\right\}_{h \in H}$ and $\left\{\hat{y}_{h, \theta_{k}}^{R W T}\right\}_{h \in H}$. In the AWGN case, these standard deviations were easily calculated by the $l^{2}$ norm of the frequency response of the corresponding filters:

$$
\sigma_{\hat{y}_{h, \theta_{k}}^{R I}}=\sigma\left\|\frac{\bar{V} G_{h, \theta}}{|V|^{2}+\varepsilon_{1}^{2}}\right\|_{2}, \quad \sigma_{\hat{y}_{h, \theta_{k}}^{R W I}}=\sigma\left\|\frac{\bar{V}|Y|^{2} G_{h, \theta}}{|V Y|^{2}+\varepsilon_{2}^{2} \sigma^{2}}\right\|_{2} .
$$

For the Poissonian case there are some modifications. The main problem is that, as the observation variance $\sigma_{z}^{2}(x)$ is not constant, the standard deviations of the directional estimates are spatially varying. It makes to compute a pointwise-varying variance for each of the estimates. Secondly, some change in the form of the Wiener denominator is required, with the constant $\sigma^{2}$ replaced by a correct estimate of the Poissonian noise power spectrum.

In order to calculate all these elements efficiently, a mixed space/frequency domain approach is exploited. Let us start from the regularized inverse stage.

\subsection{Poissonian $R I$ inverse}

The actual regularized inversion is performed in the frequency domain, and then the $L P A$ filtering is performed as a convolution of the pure regularized inverse $z^{R I}$ against the $L P A$ kernel $g_{h, \theta}$ in the spatial domain:

$$
\begin{aligned}
T^{R I}(f) & =\frac{V(-f)}{|V(f)|^{2}+\varepsilon_{1}^{2}}, & t^{R I} & =\mathcal{F}^{-1}\left(T^{R I}\right), \\
z^{R I} & =\mathcal{F}^{-1}\left(T^{R I} Z\right), & \hat{y}_{h, \theta}^{R I} & =z^{R I} \circledast g_{h, \theta} .
\end{aligned}
$$

Estimation of the standard deviation of the RI-LPA estimates (needed for the $I C I$ adaptive scale selection and for the fusing of the directional adaptive-scale estimates) is also calculated in a mixed frequency/space domain. The variance of $\hat{y}_{h, \theta}^{R I}$ is obtained as

$$
\sigma_{\hat{y}_{h, \theta}^{R I}}^{2}=\mathcal{F}^{-1}\left(\mathcal{F}\left(\left(t^{R I} \circledast g_{h, \theta}\right)^{2}\right) \cdot \Sigma_{z}^{2}\right),
$$

where $\Sigma_{z}^{2}=\mathcal{F}\left(\sigma_{z}^{2}\right)$ is the Fourier transform of the space-varying variance of $z$. Here $\sigma_{z}^{2}$ is estimated directly from the noisy observations, i.e. $\hat{\sigma}_{z}^{2}=z$ and $\Sigma_{z}^{2}=Z$. This is the simplest possible unbiased estimate of the variance, accordingly to the Poissonian rule $E\{z\}=\operatorname{var}\{z\}$.

All the varying scale estimates $\left\{\hat{y}_{h, \theta}^{R I}\right\}_{h \in H}$ obtained for each $\theta$ are fed (together with their standard deviations $\left\{\sigma_{\hat{y}_{h, \theta}^{R I}}\right\}_{h \in H}$ ) into the $I C I$ algorithm, which selects the pointwise-adaptive optimal scale $h^{+}(x, \theta)$. This is done independently for each direction $\theta$. In this way, the adaptive-scale directional estimates $\hat{y}_{h+}^{R I}\left(x, \theta_{k}\right), \theta_{k}$, $k=1, \ldots, K$, are constructed.

Fusing these directional estimates is done using the inverse variances as weights in the convex combination

$$
\begin{aligned}
& \hat{y}^{R I}(x)=\sum_{k} \lambda_{k}^{R I}(x) \hat{y}_{h^{+}\left(x, \theta_{k}\right), \theta_{k}}(x), \\
& \lambda_{k}^{R I}(x)=\sigma_{k}^{R I-2}(x) / \sum_{i} \sigma_{i}^{R I-2}(x), \\
& \sigma_{i}^{R I-2}(x)=1 / \sigma_{\hat{y}_{h^{+}}^{R I}\left(x, \theta_{i}\right), \theta_{i}}^{2}(x) .
\end{aligned}
$$

The final estimate of the $R I$ stage is the anisotropic $\hat{y}^{R I}$. The anisotropy of this estimate is a direct consequence of the adaptive selection of an optimal scale for each direction.

The use of the space domain convolutions (8) and (9) instead of multiplications in Fourier domain can speed-up calculations sig- 
nificantly, since the support of the directional $L P A$ kernels $g_{h, \theta}$ is usually very small. Moreover, this choice allows more freedom in the handling of the boundary conditions. Observe that the formula for the variance (9) can be rewritten easily in the standard convolution form $\sigma_{\hat{y}_{h, \theta}^{R I}}^{2}=\left(\mathcal{F}^{-1}\left(T^{R I} G_{h, \theta}\right)\right)^{2} \circledast \sigma_{z}^{2}$.

\subsection{Poissonian $R \boldsymbol{R} I$ inverse}

The regularized Wiener inverse algorithm proceeds similarly:

$$
\begin{aligned}
T^{R W I}(f) & =\frac{V(-f)|Y(f)|^{2}}{|V(f) Y(f)|^{2}+\varepsilon_{2}^{2} \Phi_{\eta}(f)}, & t^{R W I} & =\mathcal{F}^{-1}\left(T^{R W I}\right), \\
z^{R W I} & =\mathcal{F}^{-1}\left(T^{R W I} Z\right), & \hat{y}_{h, \theta}^{R W I} & =z^{R W I} \circledast g_{h, \theta} .
\end{aligned}
$$

Here, $\Phi_{\eta}$ is the power spectrum of the noise. It can be shown that for Poissonian observations $\Phi_{\eta}$ is constant and equal to the spatial mean of $E\{z\}$ over the image domain. As $E\{z\}=y \circledast v$ is unknown, its value may be estimated as $\hat{y}^{R I} \circledast v$. However, since $E\{\eta(x)\}=0$, we simply set $\Phi_{\eta}=$ mean $_{x}(z)$. This is an accurate approximation of $\operatorname{mean}_{x}(E\{z\})$ for large size images.

The final fused estimate of the $R I$ stage, $\hat{y}^{R I}$, is used quite naturally as a "pilot" estimate in the Wiener filtering. It means that $|Y|^{2}$ in (11) is replaced by $\left|\hat{Y}^{R I}\right|^{2}$.

Similarly to the regularized inverse stage, also the standard deviations of the RWI-LPA estimates are calculated in mixed frequency/space domain. Again, the variance of $\hat{y}_{h, \theta}^{R W I}$ is obtained as

$$
\sigma_{\hat{y}_{h, \theta}^{R W I}}^{2}=\mathcal{F}^{-1}\left(\mathcal{F}\left(\left(t^{R W I} \circledast g_{h, \theta}\right)^{2}\right) \cdot \Sigma_{z}^{2}\right) .
$$

In this second stage, $\sigma_{z}^{2}$ is estimated more accurately than in the previous one (in order to get a better estimate for $\Sigma_{z}^{2}$ ), from the regularized inverse estimate: $\hat{\sigma}_{z}^{2}=\hat{y}^{R I} \circledast v \simeq y \circledast v=\sigma_{z}^{2}$. Then, the ICI rule selects the pointwise-adaptive-scale estimate $\hat{y}_{h^{+}(x, \theta), \theta}^{R W I}(x)$, for every $x$, and for each specified direction $\theta$.

The fusing procedure is performed exactly as for the $R I$, with

$$
\begin{gathered}
\hat{y}^{R W I}(x)=\sum_{k} \lambda_{k}^{R W I}(x) \hat{y}_{h^{+}\left(x, \theta_{k}\right), \theta_{k}}(x), \\
\lambda_{k}^{R W I}(x)=\sigma_{k}^{R W I}-2(x) / \sum_{i} \sigma_{i}^{R W I}-2(x), \text { and } \sigma_{i}^{R W I}-2(x)=1 / \sigma_{\hat{y}_{h+}^{R W I}}^{2} \quad(x) .
\end{gathered}
$$

The final output of the two-stage Poissonian RI-RWI is the anisotropic adaptive estimate $\hat{y}^{R W I}$.

\subsection{Comments}

In general, the regularized inverse and regularized Wiener inverse are linear filters which actually are not appropriate to the problem with the varying signal dependent observation variance. In particular, even the ideal Wiener filter, which is obtained by setting $\varepsilon_{2}^{2}=1$ in (11) and by using the "oracle" estimates for $|Y|$ and $\Phi_{\eta}$, achieves quite a poor performance, as shown in Figure 3(right). Main reason is that the Wiener filter itself is not able to produce a global estimate fitting nonstationary varying-variance observations. However, the directional $R I$ and $R W I$ filters generate sets of estimates rich enough to select from, and the $I C I$ efficiently performs this adaptive selection.

This scale selection produces an important stabilizing effect for the algorithm overall. Indeed in (3) we assume a locally constant model for $y \circledast v$. The $I C I$ rule enables this hypothesis because the zero order $L P A$ for $y$ means that this signal is nearly constant in the adaptive-size window $w_{h^{+}}$. In this way, the adaptive scale selection allows to replace the nonlinear estimate by a switching set of linear ones of different scales.
The anisotropic fusing (10) of these adaptive estimates for various directions yields a remarkable improvement in the restoration $[4,9]$. Experiments show that the zero-order hypothesis can be relaxed and higher order filters $g_{h}$ can be more efficient.

The presented algorithm can be modified further, so to be used for restoration from signal-dependant noises other than the Poissonian one. Moreover, if the randomness of the noise is particularly high, the first stage can be executed once or more times again in order to refine the estimate of $\sigma_{z}^{2}$ by using a feedback mechanism similar to the one recently exploited for adaptation of the variance for photon-limited denoising in [10].

\section{NUMERICAL EXPERIMENTS}

In our simulations, in order to achieve a desired level of randomness (i.e. desired $S N R$ ) in the noisy Poissonian observations, we first multiply the true signal $y^{T R U E}$ (which has range $[0,1]$ ) by a scaling factor $\chi>0: y=\chi \cdot y^{T R U E}, z \sim \mathcal{P}(y \circledast v)$. Thus, $E\{z\}=\sigma_{z}^{2}=\chi \cdot y^{\text {TRUE }} \circledast v$, and $E\{z\} / \operatorname{std}\{z\}=\sqrt{\chi} \sqrt{y^{\text {TRUE }} \circledast v}$, i.e. better $B S N R$ ( $S N R$ of the blurred observation against its expectation) corresponds to larger $\chi$.

We present a "translation" to the Poissonian case of a common deblurring experiment considered by many authors for the Gaussian case (e.g. [8], [9], and references therein), where the Cameraman image is heavily blurred by a $9 \times 9$ "boxcar" uniform PSF. The PSF $v$ is assumed to be known. We create a noisy Poissonian distributed observation with a $B S N R=32.5 \mathrm{~dB}$. It corresponds to selecting $\chi=17600$. Despite such a large value of $\chi$, the nonuniformity of the noise is still a quite an essential issue for the Poisson deblurring, as the following simulations show. The actual values of the standard deviations $\sigma_{z}$ are in the range of $[0,0.0075]$ (assuming that the image is renormalized back to the range $[0,1]$ ). It is interesting to note that this level of randomness is as much as what can be observed in images taken with a consumer-level $\mathrm{CMOS}^{1}$ sensor under normal light conditions.

The proposed $R I-R W I$ adaptive algorithm is implemented with the following parameters. As in [9], a set of eight directions, $\left\{\theta_{k}\right\}_{k=1}^{8}=\{0, \pi / 4, \pi / 2, \ldots, 7 / 4 \pi\}$ and five scales, $\# H=5$, are used. Function estimation kernels were designed on conicallysupported windows choosing the first and zero $L P A$ orders for the $R I$ and $R W I$ stages, respectively. For smaller scales in $H$ the kernel support is a 1-pixel-width line. The $I C I$ thresholds and regularization parameters for the $R I$ and $R W I$, are $\Gamma_{\mathrm{RI}}=1.5, \Gamma_{\mathrm{RWI}}=1.4$, $\varepsilon_{1}=0.03$ and $\varepsilon_{2}=0.28$.

Figure 2 shows details of the blurred Poisson noisy observation and the reconstructed Cameraman image. The reconstruction is visually quite good, with most of the details properly restored and no significant distortions. The objective values of ISNR and $R M S E$ are given in Table 1. Figure 4 shows the adaptive scales selected by the $I C I$ for a vertical direction from the $R I$ and a horizontal direction from the $R W I$ stage of the algorithm. It is remarkable how these scales reveal the features of the image across the corresponding direction.

To demonstrate the significant improvement arising from our modified algorithm, we compare it against the standard Gaussian version [9]. First, we restore the image applying the algorithm in a straightforward manner, estimating the noise using a $M A D$ estimator (it gives constant $\hat{\sigma}=0.0045$ ), and using the standard parameters that were optimized for the Gaussian case. Second, we tune the parameters, in order to compensate to the wrong noise model

\footnotetext{
${ }^{1}$ Raw data from Nokia 6600 camera phone.
} 


\begin{tabular}{|l|c|c|}
\hline Algorithm & $I S N R$ & $R M S E$ \\
\hline \hline Poissonian LPA-ICI RI-RWI & $\mathbf{6 . 6 1}$ & $\mathbf{0 . 0 4 2 8}$ \\
\hline Optimized Gaussian LPA-ICI RI-RWI & 6.03 & 0.0458 \\
\hline Gaussian LPA-ICI RI-RWI [9] & 5.38 & 0.0493 \\
\hline
\end{tabular}

Table 1. MSE and $I S N R(\mathrm{~dB})$ for Cameraman image for Poisson image reconstruction.
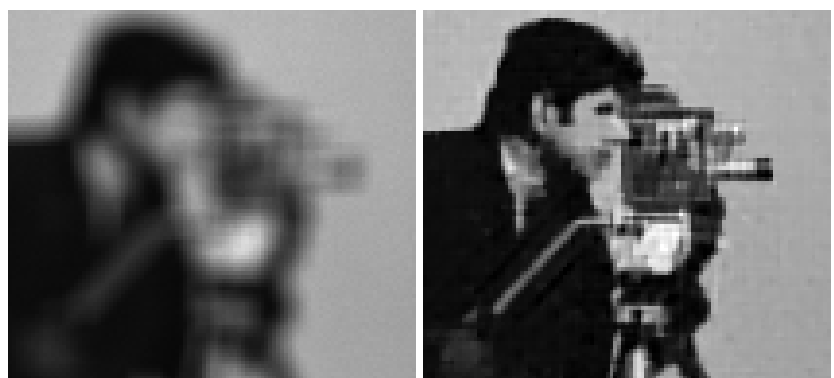

Fig. 2. Deblurring the Cameraman image. Left is a fragment of the noisy blurred observation ( $B S N R=32.5 \mathrm{~dB})$. Right is the reconstructed image obtained by the proposed Poissonian adaptive deconvolution algorithm, $I S N R=6.61 \mathrm{~dB}$.
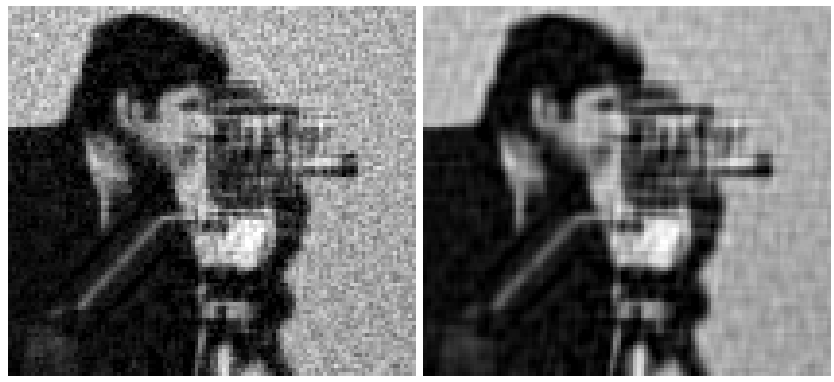

Fig. 3. Result of the pure regularized inverse $z^{R I}$ from (8) (left) and the "oracle" Wiener estimate, $I S N R=5.22 \mathrm{~dB}$ (right).

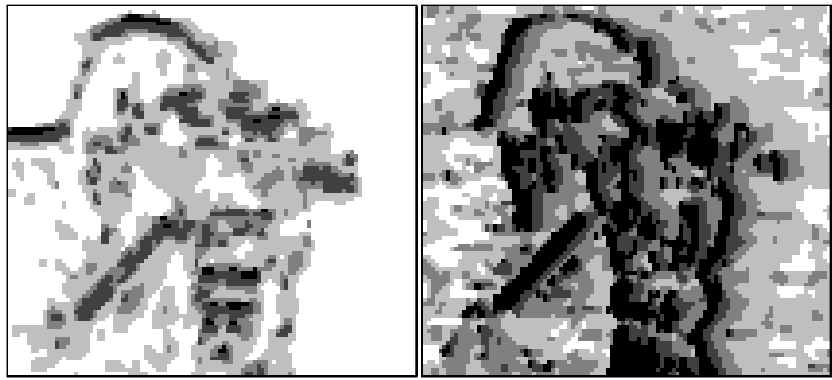

Fig. 4. Adaptive scales: $R I h^{+}(\cdot, \pi / 4)$ (left), and $R W I h^{+}(\cdot, 0)$ (right). Darker color represents smaller scales.

assumed by algorithm, trying to obtain the best possible restoration. Results are shown in the Table and in Figure 5. Numerically, both results obtained by the Gaussian algorithms are worse than the one obtained with the algorithm specifically designed for the Poissonian data. Comparing images in Figure 5 we may note the enhancement obtained by the parameter optimization. A further comparison with the reconstructed image in Figure 2(right) obtained by the algorithm developed for the Poissonian data demonstrates an obvious visual advantage of the proposed algorithm.
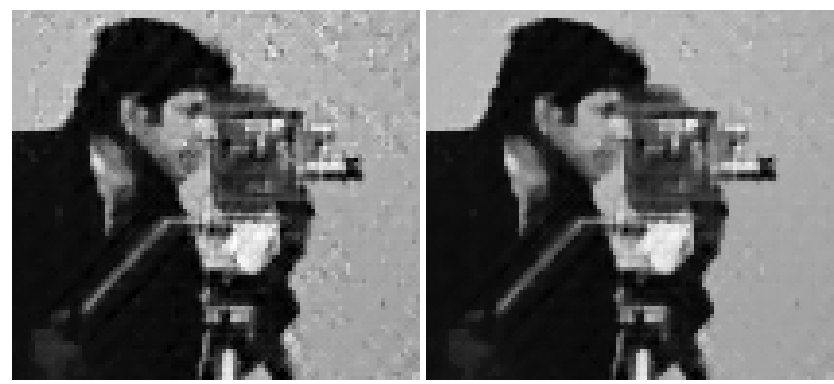

Fig. 5. Filtering the Poisson data with the algorithm developed for the Gaussian one. Standard selection of the algorithm parameters gives a poor estimate, $I S N R=5.38 \mathrm{~dB}$ (left). Up to some extent, it can be improved by manually optimizing some algorithm parameters, $I S N R=6.03 \mathrm{~dB}$ (right).

\section{REFERENCES}

[1] Bertero, M., and P. Boccacci, Introduction to inverse problems in imaging. Inst. of Physics Publishing, 1998.

[2] Fan, J., and I. Gijbels, Local polynomial modelling and its application. Chapman and Hall, 1996.

[3] Fienup, J., D. Griffith, L. Harrington, A.M. Kowalczyk, J.J. Miller, and J.A. Mooney, "Comparison of reconstruction algorithms for images from sparse-aperature systems", Proc. SPIE 4792-01, July 2002.

[4] Foi, A., V. Katkovnik, K. Egiazarian, and J. Astola, "A novel anisotropic local polynomial estimator based on directional multiscale optimizations", Proc. 6th IMA Int. Conf. Math. in Signal Processing, Cirencester (UK), pp.79-82, 2004.

[5] Foi, A., V. Katkovnik, K. Egiazarian, and J. Astola, "Inverse halftoning based on the anisotropic LPA-ICI deconvolution", Proc. of SMMSP 2004, pp. 49-56, 2004.

[6] Goldenshluger, A., and A. Nemirovski, "On spatial adaptive estimation of nonparametric regression", Math. Meth. Statistics, vol. 6, pp. 135-170, 1997.

[7] Katkovnik, V., "A new method for varying adaptive bandwidth selection", IEEE Trans. on Signal Processing, vol. 47, no. 9, pp. 2567-2571, 1999.

[8] Katkovnik, V., K. Egiazarian, and J. Astola, "A spatially adaptive nonparametric regression image deblurring", IEEE Trans. on Image Processing, in print, 2004.

[9] Katkovnik, V., A. Foi, K. Egiazarian, and J. Astola, "Directional varying scale approximations for anisotropic signal processing," Proc. of EUSIPCO 2004, pp. 101-104, 2004.

[10] Katkovnik, V., A. Foi, K. Egiazarian, and J. Astola, "Anisotropic local likelihood approximations", Proc. of Electronic Imaging 2005, 5672-19, 2005.

[11] Kondo, K., Y. Ichioka, and T. Suzuki, "Image restoration by Wiener filtering in presence of signal-dependent noise", $A p$ plied Optics, vol. 16, N. 9, 1977.

[12] Rooms, F., W. Philips, and P. Van Oostveldt, "Integrated approach for estimation and restoration of photon-limited images based on steerable pyramids", Proc. of EC-VIP-MC 2003, pp. 131-136, 2003.

[13] Wach, H.B., and E.R. Dowski Jr., "Noise modeling for design and simulation of color imaging systems", Proc. of IS\&T/SID's 12th Color Imaging Conference, CIC 12, 2004.

[14] White, R.L., "Image restoration using the damped Richardson-Lucy method", Astr. Data An. Software and Systems, ASP Conf. Series, vol.61, pp. 292-295, 1994. 